\title{
FTR allocations to ease transition to nodal pricing: An application to the German power system ${ }^{2}$
}

\author{
Friedrich Kunz $^{\mathrm{a}, 1}$, Karsten Neuhoff ${ }^{\mathrm{b}, \mathrm{c}, 2}$, Juan Rosellón ${ }^{\mathrm{a}, \mathrm{d}, \mathrm{e}, *, 3}$ \\ a DIW Berlin, Department of Energy, Transportation, Environment, Mohrenstraße 58, 10117 Berlin, Germany \\ b DIW Berlin, Department of Climate Policy, Mohrenstraße 58, 10117 Berlin, Germany \\ c Technical University Berlin, Germany \\ d CIDE, Department of Economics, Carretera México-Toluca 3655 Col. Lomas de Santa Fe, 01210 Mexico City, Mexico \\ e Universidad Panamericana, Campus Mexico, Escuela de Ciencias Económicas y Empresariales, Mexico City, Mexico
}

\section{A R T I C L E I N F O}

\section{Article history:}

Received 29 June 2015

Received in revised form 9 September 2016

Accepted 17 September 2016

Available online 23 September 2016

\section{JEL classification:}

L50

L94

Q40

\section{Keywords:}

Financial transmission rights

Nodal prices

Congestion management

Electricity

Germany

\begin{abstract}
A B S T R A C T
A shift from zonal to nodal pricing improves the efficiency of system operation. However, resulting price changes also shift surplus across generation and loads at different locations. As individual actors can lose, they might oppose any reform. We explore how allocation of financial transmission rights can be used to mitigate the distributional impact. The fundamental effects with regard to reference node/hub for FTRs, the share of FTRs to be freely allocated and the metric to determine the proportion of rights allocated are explored. We test the results in a setting based on the hourly modeling of the German power system at nodal representation.
\end{abstract}

(C) 2016 The Authors. Published by Elsevier B.V. This is an open access article under the CC BY-NC-ND license (http://creativecommons.org/licenses/by-nc-nd/4.0/).

\section{Introduction}

It is not just academics accepting that nodal pricing is ultimately the most secure and efficient way of operating a power system, but also policy makers in many countries. However, one major obstacle for implementing nodal prices, or the definition of smaller pricing zones,

it A previous version of this paper was presented at the Conference on Energy Industry at a Crossroads: Preparing the Low Carbon Future/TIGER Forum, Toulouse, 5-6 June, 2014. We are grateful for the valuable feedback from Shmuel Oren, Thomas-Olivier Léautier, and Seabron Adamson.

* Corresponding author at: DIW Berlin, Department of Energy, Transportation, Environment, Mohrenstraße 58, 10117 Berlin, Germany.

E-mail addresses: fkunz@diw.de (F. Kunz),kneuhoff@diw.de (K. Neuhoff), juan.rosellon@cide.edu, jrosellon@diw.de (J. Rosellón).

URL: http://www.up.edu.mx (J. Rosellón).

${ }^{1}$ Friedrich Kunz acknowledges financial support by the Mercator foundation within the project MASMIE.

2 Karsten Neuhoff acknowledges financial support from the BMWi (German Federal Ministry for Economic Affairs and Energy) funded projects SEEE (funding number 03MAP316) and EUREEM (funding number 03MAP274).

3 Juan Rosellón acknowledges support from a Marie Curie International Incoming Fellowship within the 7th European Community Framework Programme, as well as from Conacyt (Mexican Science Foundation, p. 131175) and a FAI grant from CIDE. is their implied distributional impacts. For instance, generators in low-price and loads in higher-price zones might lose out with the new pricing system. Thus, a central element for the successful implementation of nodal pricing is the parallel allocation of financial hedges, such as financial transmission rights (FTRs). ${ }^{4}$ These are effectively used in the electricity markets of the US Northeast, among other places. This process has allowed (i) to compensate losers, and (ii) to reduce the risk for players since they are protected against potential price impacts. The latter point is particularly relevant since most market participants

\footnotetext{
${ }^{4}$ A FTR is defined according to: i) an injection node and a withdrawal node; ii) a megawatt (MW) award that remains invariable for the length of the contract; and iii) a life term. FTRs might be obligations or options (see Rosellón and Kristiansen, 2013). With an obligation, the holder has the right to collect payment (when congestion takes place) or the requirement to pay (when congestion in network occurs in the opposite direction to originally defined). The payment is determined by the difference in prices between the injection node and the withdrawal node multiplied by the contractual MW amount. FTR options grant only the non-negative gains to its owner since there is no charge when congestion occurs in the opposite direction. FTRs are usually allocated through auctions run by an independent system operator (ISO) that ensure that only feasible FTRs are issued, so that transmission flows do not violate capacity constraints in a power-flow model. For FTR obligations, Hogan (1992) and Hogan (2013) show that revenue from congestion rents in the spot market is enough to cover all payments in the FTR contracts (i.e, simultaneous technical feasibility implies revenue adequacy).
} 
are risk averse, particularly toward risks that are partially attributed to regulators, like a power-market design choice. Such risks are naturally difficult to assess.

FTRs started being implemented during the past two decades. The technical characteristics of an electricity grid (as represented by the Kirchhoff's laws) led economists to create markets for transmission property rights (Hogan, 1992). FTR markets were implemented in the northeast US power markets in the late 1990s. In other countries, there were intense discussions on the need for congestion hedging from transmission price risk, such as in the case of New Zealand, where nodal prices were implemented as early as 1989. Notwithstanding, the initial allocation of FTRs in a newly created nodal-price system has, in particular, been one of the most highly disputed facets of market liberalization processes in various countries. After all, an initial provision of FTRs is an intricate process since it boils down to sharing the congestion rents among the various market participants. ${ }^{5}$

In this paper, we are interested in the process of initial allocation of FTRs in a recently restructured market. In the United States, for instance, the early implementation of an FTR market in the NYISO had to address "grandfathered" contracts. That is, long-term hedging contracts already in place, based on previous transmission capacity rights of utilities, that had to be respected during the allocation of new FTRs within transformation of the system to nodal prices. ${ }^{6}$ In New Zealand, the adoption of nodal prices dates back to 1989 , but hedging contracts were not immediately implemented at that time. It was not until late past decade (NZEA, 2010) that discussions on FTR implementation took place both at the inter- and intra-regional levels. ${ }^{7}$ In Australia, a zonal pricing system with a complicated initial allocation of FTRs was developed. There are continuing discussions in Australia to change to a fully nodalpricing scheme, which is proven to support FTR contracts more firmly, but the implied sharing of rents among economic agents remains a serious obstacle. ${ }^{8}$

In Europe, transmission rights between pricing zones had been grandfathered in the form of physical transmission rights between pricing zones (usually countries) to match commercial contractual arrangements at the time of liberalization of the power markets. These long-term transmission rights have been largely phased out and, as of 2014 , most transmission rights between pricing zones are auctioned for a year or other shorter time periods and transmission capacity is allocated in implicit auctions in day-ahead markets. Financial transmission rights are only present in the Nordic area, but are exclusively bilateral contractual arrangements with limited liquidity as the transmission system operator does not issue transmission rights backed by congestion revenue like in other markets. De Maere d'Aertrycke and Smeers (2013) further analyze the potential introduction of FTRs in the European market-coupling system (MC) and show with various examples that FTRs would not lead to simultaneous feasibility and, hence, would not be revenue adequate. That is, the zonal-pricing nature of the MC system, together with the lack of real-time markets,

\footnotetext{
${ }^{5}$ One proven advantage, however, has been that through the introduction of nodal pricing in liberalized markets, the overall level of network utilization increases and therefore also the volume of FTRs that are available for allocation.

${ }^{6}$ Adamson and Parker (2013) analyze the efficiency implications of the FTR auction system in the NYISO market, and conclude that, after initial relative inefficiency, market participants learned the rules, such that the forward-looking allocative efficiency of the NYISO's FTR market design is now robust.

7 According to Read and Jackson (2013), such a long delay is partly explained by the special characteristics of the New Zealand's market, which is dominated by hydro generation, a sparse network, and where reserve support is typically more important than network capacity constraints. There is also a "tidal-flow" nature of the New Zealander electricity system, so that south-to-north flows occur in wet years and north-to-south flows in dry years. This complicates the use of FTR obligations (Read, 2009).

${ }^{8}$ Read (2007) proposes an intermediate alternative where all Australian market participants (generators, loads, network capacity and ancillary-service providers) are selectively exposed to nodal pricing, but provided with FTRs to hedge the risk implied. Given the many vested interests involved, the major implementation obstacle to such a proposal is how congestion rents would be allocated among market participants, such as between generators and interconnector flows, as well as between new and old market players.
}

lead to lack of firmness of FTRs and thus make the ACER's foreseen transmission-right framework guidelines unlikely.

In this paper, we explore a different (but related) issue to the one discussed in De Maere d'Aertrycke and Smeers (2013). Namely, we develop modeling frameworks to explore how an initial free allocation of FTRs at the time of a transition from uniform to nodal pricing could be designed so as to limit revenue or cost changes for generation and load. We rely on two basic modeling frameworks. In our reference uniform-pricing framework, generators bid prices and quantities which are assumed to represent true costs and available capacity. The market is then cleared in merit order, ignoring transmission constraints and all dispatched capacity is paid a uniform price equal to the market clearing price for the quantities cleared by the auction. Congestion resulting from the dispatch is relieved by the system operator through least cost re-dispatch at marginal generation costs and the load pays the auction uniform clearing price plus an uplift socializing the total cost of congestion relief.

In a second LMP-based market framework, the auction clears based on transmission constrained least cost dispatch. Generators receive and load pays locational marginal prices for the produced and consumed energy. Congestion rents together with costs of transmission systems are allocated to the load on a prorate basis. The second proposed approach treats the uniform pricing mechanism as a reference position and uses FTRs to avoid deviations of costs or revenues linked to locational specific prices that could trigger political opposition while achieving market efficiency by transitioning to a nodal pricing based dispatch.

FTRs are defined as swaps between respective nodes and trading hubs where the price is the load weighted nodal prices at load nodes. The FTRs are allocated initially for free to generators and loads at the respective nodes corresponding to each particular FTR subject to simultaneous feasibility constraints limiting the issue of FTRs. FTRs allocated to generation at high price nodes or load at low price nodes are likely to exhibit in expectation a negative value, while FTRs allocated to generation at low price nodes and load at high price nodes will have in expectation a positive value.

To illustrate the basic dynamics of these two above frameworks, we firstly apply them to a simplified three-node network, and then we obtain simulated results for the German power system so as to quantify the merits of different FTR allocation approaches. In all approaches, the allocation remains independent from subsequent operational choices by generation and load so as to avoid incentives to distort operation to impact allocation of FTRs. The modeling effort is focused on the distributional aspects, and does not quantify the impact on efficiency savings or system security. The document explores the idea of awarding point-to-point (or point-to-hub) FTRs as a mechanism for offsetting the distributional consequences resulting from a transition from a uniform pricing approach that hides the real cost or value of electricity to the system at different locations (e.g. Germany) to a locational marginal pricing (LMP) design that correctly prices electricity at according to system cost or benefit (e.g. NYISO). The point is that such a compensation scheme facilitates transition by smoothing the distributional impact that may be somewhat uncertain ex-ante and therefore of particular concern for risk averse market participants. Such transfers might be the main source of resistance to a nodal pricing regime, and by addressing these objections it would be politically possible to implement nodal pricing, and gain the efficiency benefits and enhanced system operation security of that design.

In our three-node network, we address the optimal allocation of FTRs-assuming that the regulator has full information across the entire system-so as to investigate the extent that the FTR allocation may reduce distributional impacts. However, in practice, it might be difficult to replicate an optimal allocation scheme that, for example, could minimize the distributional impacts for changing from a uniformpricing system to a nodal one. In particular, how could a European regulator agree on the necessary parameterization for a corresponding EU power system model that would be the basis for such an optimal 
FTR-allocation approach? Thus, next we consider simplified FTR allocation methods that could be available in practice and simulate the results to compare across such allocation methods. More specifically, we propose and compare two alternative FTR allocation criteria: one based on previous generation capacity and load consumption, another based on historic generation and load in uniform pricing regime. We illustrate the effects of each approach for a three-node stylized network, and then carry a simulation study using an elaborate network representation of the German electricity system.

The rest of the paper is organized as follows. In Section 2, we present our models for uniform pricing, nodal pricing and for the optimal allocation of FTRs. In Section 3, we compare, in a three-node network, how different FTR allocation methods function; while in Section 4, we do the same thing using real data from the German power market. Section 5 concludes.

\section{Optimization models}

For our simulations, we specify three optimization models. The underlying nomenclature of indices, parameters and variables is given in Appendix A. The first model characterizes the current German electricity market clearing approach, with a uniformly priced national spot market fully abstracting from physical transmission limits and a subsequently following congestion management through curative power plant re-dispatch (Kunz, 2013). The second model follows the idea of nodal pricing and combines the economic dispatch of power plants in the spot market and the optimal operation of the physical transmission network. Both models, the uniform and nodal pricing model, differ in the way congestion in the transmission network is handled. The uniform pricing model relies on curative methods (re-dispatch), whereas preventive congestion management is applied in the nodal pricing. Finally, we specify a third optimization model that addresses the allocation of financial transmission rights to market participants based on the results of the uniform and nodal pricing market models. Herein, the feasibility and the revenue adequacy of the FTR allocation are tested.

\subsection{Uniform pricing model}

\subsubsection{Market clearing}

The uniform pricing model starts with the optimization of the generation dispatch (Eq. (1)) subject solely to the energy balance of demand and supply (Eq. (2)), and the maximum generation restriction (Eq. (3)). Thus, restrictions stemming from limited transmission capacity of the network are not taken into account, which results in a uniform price for the entire electricity market. ${ }^{9}$

$\min _{G} \sum_{p, t} m c_{p} G_{p, t}$

$\sum_{n} d_{n, t}-\sum_{p} G_{p, t}-\sum_{n} g_{n, t}^{R E S}=0$

$0 \leq G_{p, t} \leq g_{p}^{\max }$

\subsubsection{Congestion management}

Given the optimized generation dispatch of the market clearing model, network restrictions due to limited transmission capacity are considered in the congestion management model. Herein, the generation dispatch is adjusted in order to ease overloading of transmission lines. The congestion management model minimizes the congestion management cost consisting of upward $G_{p}^{U P}$ and downward regulation $G_{p}^{D O W N}$ of generation enumerated at their marginal cost (Eq. (4)). The approach

\footnotetext{
9 The dual of the energy-balance constraint (Eq. (2)) provides such a uniform price.
}

reflects the cost-based re-dispatch. Again, the energy balance (Eq. (5)) has to be ensured as well as maximum and minimum generation restrictions (Eqs. (6) and (7)). The parameter $g_{p}$ reflects the optimized generation of the market clearing model. Finally, the feasibility of the transmission flows is ensured in Eqs. (8) and (9) using a lossless DC load flow approach (Leuthold et al., 2012).

$$
\begin{aligned}
& \min _{G^{U P}, G^{D O W N}, \Delta} \sum_{p, t} m c_{p}\left(G_{p, t}^{U P}-G_{p, t}^{D O W N}\right) \\
& d_{n, t}-\sum_{p \in A(n)}\left(g_{p, t}+G_{p, t}^{U P}-G_{p, t}^{D O W N}\right)-g_{n, t}^{R E S}-\sum_{n n} b_{n, n n} \Delta_{n, t}=0 \\
& 0 \leq G_{p, t}^{U P} \leq g_{p}^{\max }-g_{p, t} \\
& 0 \leq G_{p, t}^{\text {DOWN }} \leq g_{p, t}
\end{aligned}
$$$$
\left|\sum_{l} h_{l, n} \Delta_{n, t}\right| \leq p_{l}^{\max }
$$$$
\Delta_{n^{\prime}, t}=0
$$

\subsection{Nodal pricing}

In contrast to the uniform pricing model, the nodal pricing model combines the optimization of the generation dispatch and the transmission usage. Thus, the previously specified market clearing model of the uniform pricing model is extended by transmission network restrictions (Eqs. (8) and (9)) to form a nodal pricing approach. The model minimizes generation costs (Eq. (10)) subject to the nodal energy balance (Eq. (11)), generation capacity limitations (Eq. (3)), and DC load flow restrictions (Eqs. (8) and (9)). Again, losses stemming from transmission flows are neglected. ${ }^{10}$

$\min _{G, \Delta} \sum_{p, t} m c_{p} G_{p, t}$

$d_{n, t}-\sum_{p \in A(n)} G_{p, t}-g_{n, t}^{R E S}-\sum_{n n} b_{n, n n} \Delta_{n, t}=0$

\subsection{FTR allocation}

In the following, we define two approaches, volume- and capacitybased, for an initial allocation of FTR obligations. We define a reference bus, as demand weighted average of all nodal prices, thus following the typical definition of trading hubs in US systems. FTRs are issued from generation nodes to the reference bus and from the reference bus to nodes with load. As any trading relation can be hedged with such a pair of FTRs, point-to-point FTRs are not necessary and not considered.

As we focus on the distributional effects, we do not model any re-trading of FTRs which would be motivated by market participants that aim to more closely hedge exposure to congestion costs with FTRs. Such re-trading, would under the assumption of perfect competition, not have a distributional impact.

The first approach allocates FTRs to conventional $\left(F T R_{p}^{G}\right)$ and renewable $\left(F T R_{n}^{R E S}\right)$ generators based on historical production ${ }^{11}$

\footnotetext{
10 The dual of the energy-balance constraint (Eq. (11)) provides the nodal prices. 11 Historical production refers to generation quantities realized under uniform pricing over a specified time horizon from the past. Therefore, it is assumed that market participants cannot alter their generation in order to impact the FTR allocation. In the following calculations, we use the generation quantities of the uniform pricing setting for the FTR allocation.
} 
(volume-based approach), whereas the second approach relies on installed generation capacities (capacity-based approach) to determine the amount of FTRs. These measures are applied for the allocation of FTRs to conventional and renewable generation. On the demand side, FTRs ( $F T R_{n}^{D}$ ) are allocated relative to consumption, given the total amount of FTRs allocated to generation. For both allocation approaches, we explore different levels or amounts of total FTRs, ranging from $0 \%$ to $100 \%$ of historical generation or installed capacity, respectively.

For each allocation approach, the feasibility as well as the revenue adequacy is checked. Feasibility means that FTR allocation is possible with the underlying physical transmission network and its capacities (Eqs. (8), (9), (12), and (13)). On the other hand, revenue adequacy requires that the payments resulting from FTR obligations do not exceed the congestion rent $c r_{t}^{T S O}$ earned by the TSO (Eq. (14)). If the initial allocation is feasible in these terms, the implications for the market participant's surplus are quantified as additional revenues stemming from holding an FTR due to nodal price differences. The price at the reference bus price $_{\text {slack, } t}$ where all FTRs are related to is defined as the demand weighted average of all nodal prices.

$\sum_{n} F T R_{n}^{D}-\sum_{n} F T R_{n}^{R E S}-\sum_{p} F T R_{p}^{G}=0$

$F T R_{n}^{D}-F T R_{n}^{R E S}-\sum_{p \in A(n)} F T R_{p}^{G}-\sum_{n n} b_{n, n n} \Delta_{n, t}=0$

$\begin{aligned} \operatorname{cr}_{t}^{T S O} & -\sum_{p}\left(\text { price }_{\text {slack }, t}-\text { price }_{A(n), t}\right) F T R_{p}^{G}-\sum_{n}\left(\text { price }_{\text {slack }, t}-\text { price }_{n, t}\right) F T R_{n}^{R E S} \\ & -\sum_{n}\left(\text { price }_{n, t}-\text { price }_{\text {slack }, t}\right) F T R_{n}^{D}=0\end{aligned}$

\section{FTR allocation in a three-node network}

In a first step, we consider a simple three-node network setting in order to examine the implications of the different initial FTR allocation approaches on the different market participants. Generally, we consider two time steps reflecting off-peak and peak load, two conventional generators with different marginal generation cost, as well as two load locations. The underlying transmission infrastructure is characterized by equal technical characteristics and only one particular line has limited capacity. The setting is summarized in Fig. 1.

Table 1 depicts the surplus of the considered market participants for the uniform and the nodal pricing regime. Due to the different ways to manage network constraints, market prices differ and, hence, so does the surplus of load and generation. Furthermore, the transmission part faces congestion management costs with respect to the uniform pricing when shifting production from cheap to expensive generation

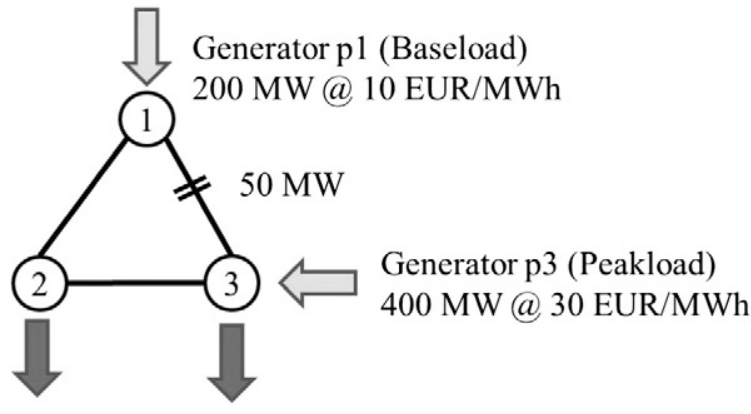
Load n2
Load n 3
t1: $50 \mathrm{MW}$
t1: $100 \mathrm{MW}$
t2: $80 \mathrm{MW}$
t2: $160 \mathrm{MW}$

Fig. 1. Three-node network setting with two time periods $(t 1, t 2)$, symmetric resistance of lines and a transmission constraint of $50 \mathrm{MW}$ on line 13.
Table 1

Summary of market participant's revenue, costs, and surplus in different pricing regimes in EUR.

\begin{tabular}{|c|c|c|c|}
\hline & $\begin{array}{l}\text { Uniform } \\
\text { pricing }\end{array}$ & $\begin{array}{l}\text { Nodal } \\
\text { pricing }\end{array}$ & $\begin{array}{l}\text { Surplus change including } \\
\text { socialized transmission surplus }\end{array}$ \\
\hline \multicolumn{4}{|l|}{ Load } \\
\hline \multicolumn{4}{|l|}{$\mathrm{n} 2$} \\
\hline Revenue & 0 & 0 & - \\
\hline Costs & 3800 & 2600 & - \\
\hline Surplus & -3800 & -2600 & +2200 \\
\hline \multicolumn{4}{|l|}{ n3 } \\
\hline Revenue & 0 & 0 & - \\
\hline Costs & 7600 & 7800 & - \\
\hline Surplus & -7600 & -7800 & +1800 \\
\hline \multicolumn{4}{|l|}{$\begin{array}{l}\text { Generation } \\
\text { p1 }\end{array}$} \\
\hline Revenue & 6150 & 2150 & - \\
\hline Costs & 2150 & 2150 & - \\
\hline Surplus & 4000 & 0 & -4000 \\
\hline \multicolumn{4}{|l|}{ p3 } \\
\hline Revenue & 5250 & 5250 & - \\
\hline Costs & 5250 & 5250 & - \\
\hline Surplus & 0 & 0 & 0 \\
\hline \multicolumn{4}{|l|}{ Transmission } \\
\hline Revenue & 1350 & 3000 & - \\
\hline Cost & 4050 & 0 & - \\
\hline Surplus & -2700 & 3000 & Socialized to load \\
\hline
\end{tabular}

assets in order to correct for violations of the transmission constraints (re-dispatch) and revenues in the nodal pricing stemming from an implicit allocation of network capacity. Further on, we assume in a first step that the transmission surplus is redistributed or socialized to load as part of the network tariffs. ${ }^{12}$ Thus, loads at nodes 1 and 2 are left with an overall benefit of 2200 EUR and 1800 EUR, respectively. On the other hand, generation $\mathrm{p} 1$ is negatively affected and faces a loss in surplus by -4000 EUR for the two considered time periods.

So far, we have considered a full redistribution of transmission surplus to load, which results in overall profits for load. In the following, we relax this assumption and apply an initial FTR allocation that allocates transmission surplus, in particular the congestion rent of the nodal pricing, to all market participants; including generators. Fig. 2 depicts the implications of an increased amount of FTRs allocated to market participants based on either their installed capacity or the historical production of the uniform pricing regime. If not all congestion rent is allocated through FTRs, the remaining rent is again socialized to load. The allocation is undertaken as long as the feasibility of the FTR allocation is ensured.

As seen in Fig. 2, both allocation approaches initiate a redistribution of congestion rents and bring down the loss or profit through a pricing change for most market participants. This is particularly true for the volume-based allocation where the loss experienced by generation is nearly compensated. On the other hand, the initial gain of load is decreased and redistributed to generation. If we instead consider an allocation of FTRs based on installed capacity, the general result remains true. The peak generator located at $\mathrm{n} 3$ obtains more FTRs with capacity based allocation than in the allocation based on historic (modeled) production. As the price at $\mathrm{n} 3$ is higher than at the reference node, the allocation of the FTR requires payments from the FTR holder, hence the declining surplus of the peak generation at $n 3$.

If we adjust the optimization problem described in Section 2.3 to minimize the absolute difference between surpluses of both pricing regimes, we can easily determine an optimal initial FTR allocation that minimizes the surplus variance. ${ }^{13}$ Within this approach, the FTR

12 The transmission surplus is socialized to load in each node according to their share on the entire load. Furthermore, the distribution of transmission surplus solely to load instead of generation or a combination of both reflects the current approach of most European countries.

13 See Appendix A for the detailed model formulation. 


\section{Capacity-based FTR allocation}
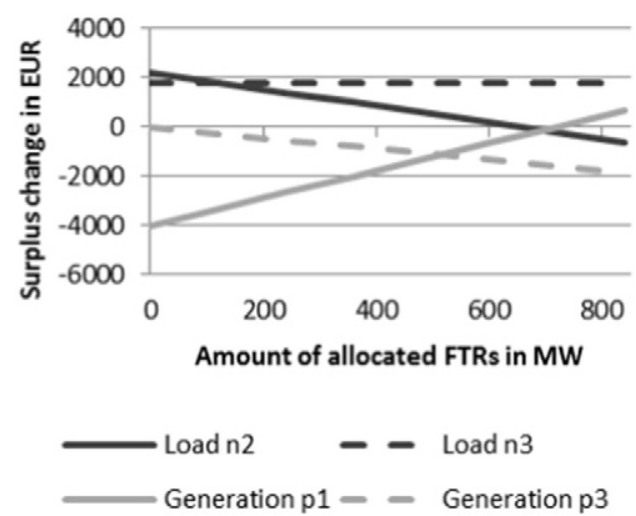

\section{Volume-based FTR allocation}
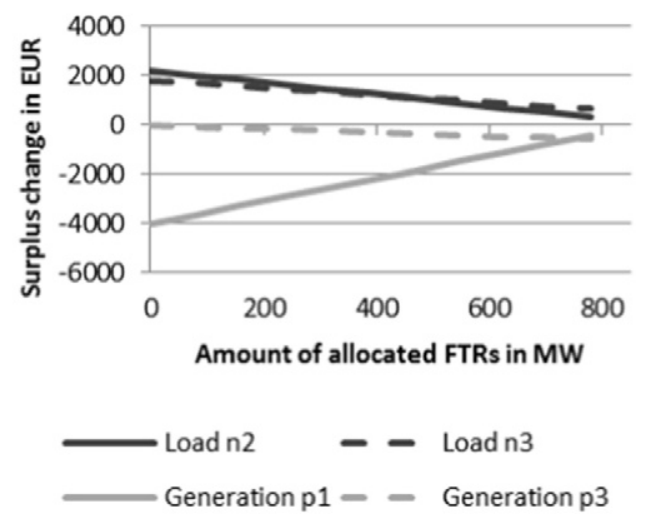

Fig. 2. Change of market participant's surplus between uniform and nodal pricing considering different initial FTR allocation regimes in the three-node setting.

allocation is not determined by capacity or production, and can be optimized with respect to the defined objective. Using this setting, an optimized FTR allocation is determined, which allocates 75 MW FTRs to generation located at node $\mathrm{n} 1$ and load located at node $\mathrm{n} 3$. All other market players do not receive an FTR. With this FTR allocation, the initial loss of generation at node $\mathrm{n} 1$ of -4000 EUR can be reduced to -1500 EUR, while the significant positive surplus of load, taking the socialized congestion rent into account, is reduced.

An intriguing result from the exercise was for us, the choice of the reference point for financial contracts matters. In order to avoid the need to issue FTRs between each pair of nodes, typically a reference node or weighted set of nodes (reference hub) is defined. Market participants thus require only FTRs to and from each node to the hub. Market participants with a FTR from the origin of a power transfer to the hub, and a subsequent FTR from the hub to the destination, are indifferent to the definition of the hub. However, if the initial free FTR allocation is for contracts from the location of load/generation to the reference location (node or hub) only, then the definition of the hub matters. To minimize the effect, the reference location of FTRs should reflect average rather than extreme prices and location (see Olmos and Neuhoff (2006) for a similar calculation at European level).

Real networks, generation and load patterns are obviously far more complex than our three-node example. The large number of nodes and linkages could-in principle-moderate the impact of any one constraint, and thus reduce some of the large variations that are demonstrated for the three-node network, thus enhancing the value of FTRs to address distributional impacts of nodal pricing implementation. On the contrary, the specific circumstances of nodes, generation and load could reduce the value of using FTRs to address distributional impacts. To assess this trade-off, we quantify the role of FTR allocation proportional to annual generation/load and proportional to installed generation capacity/peak load in the following application to the German power system.

\section{FTR allocation for the German power system}

In a second analysis, we apply the described methodology to a dataset reflecting the 2012 German power system. A detailed description of the dataset and the underlying data sources is provided in Egerer et al. (2014). ${ }^{14}$ The dataset covers an entire year, but we focus on three characteristic weeks to limit computational requirements. The weeks are selected due to their different load and renewable generation pattern: one covering a summer week with high solar generation and two covering winter weeks that are characterized by high load and low or high wind generation, respectively. For these three weeks, we

\footnotetext{
14 The original dataset and a basic formulation of the underlying ELMOD model can be derived from http://www.diw.de/elmod.
}

determine the uniform and nodal pricing solution, both for the off-peak and the peak periods, as well as apply the different FTR allocation approaches.

In the following, the implications on load, conventional and renewable generation are analyzed in detail. First, the absolute changes in surplus between nodal and uniform pricing are shown in Fig. 3 for the three analyzed weeks. The transmission congestion costs occurring in the uniform pricing due to necessary re-dispatch of generation as well as the transmission congestion rent of the nodal pricing are socialized to load as in the three-node setting. Thus, they are implicitly considered in the results and, therefore, not listed explicitly in the following graphs.

As seen in Fig. 3, the changes are significant in winter weeks with a significant amount of network congestion. In the summer weeks, the congestion is low and therefore both pricing regimes yield nearly identical results. In particular, demand wins in all three cases from a switch to nodal pricing as, firstly, the price level decreases due to explicit pricing of network congestion and, secondly, the socialization of congestion rents in the nodal pricing regime to electrical load. On the other hand, the lower price level decreases the generation revenues in all three cases. In particular, the last case, with high wind generation in the winter week, indicates greater losses for renewable generation than other cases. Due to high wind generation in the northern part of Germany, significant congestion occurs on the north-south corridor resulting in lower prices in the northern part of Germany and higher prices in the southern part compared to the uniform pricing. As most of the conventional as well as renewable generation is located in the northern part, this results in a negative effect of nodal pricing for generation. Furthermore, the congestion and, henceforth, congestion rents

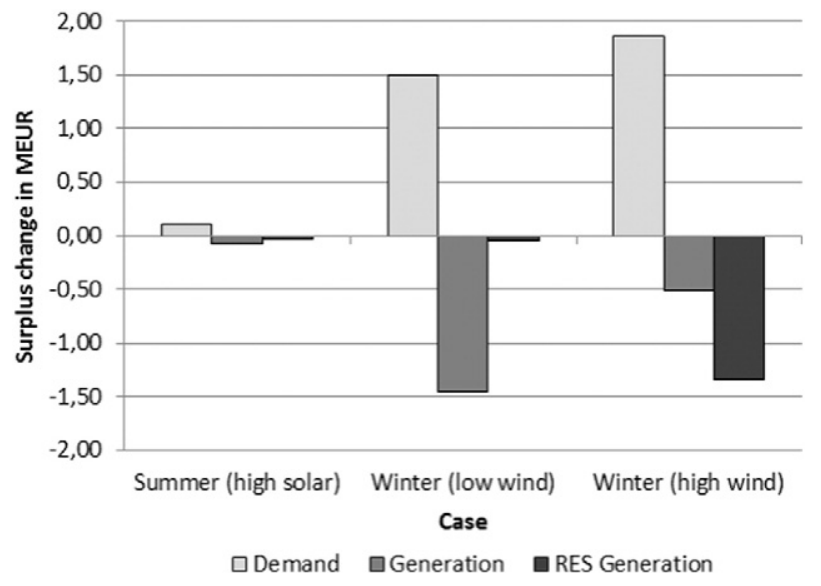

Fig. 3. Change of surplus between uniform and nodal pricing. 


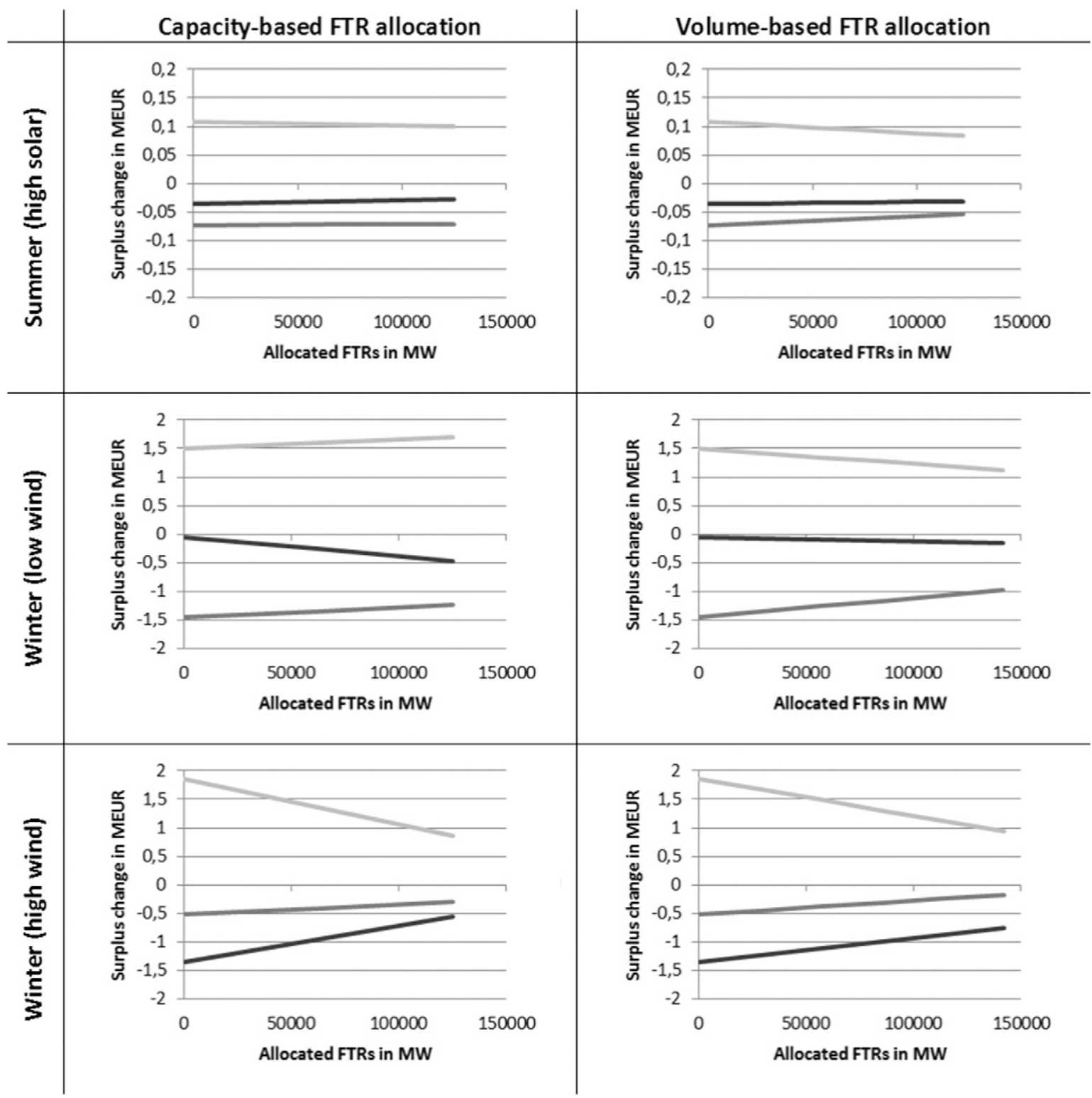

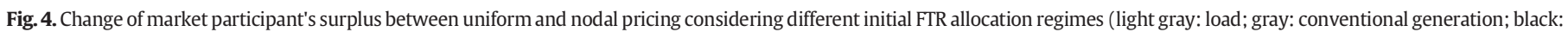
renewable generation).

strengthen the position of the demand resulting finally in profits compared to the uniform pricing regime. It is important to note that the level of congestion in the German power system is relatively low. For instance, in the winter week with high wind generation, congestion rent in the nodal pricing amounts to $1.6 \mathrm{mEUR}$ or $0.2 \%$ of electricity cost for demand. ${ }^{15}$

Comparing the different FTR allocation approaches, Fig. 4 depicts the surplus changes for the capacity-based (left side) and the volume-based allocation approach (right side) for the three weeks considered. Furthermore, each graph visualizes the implications for an increasing amount of FTRs to be allocated as long as the FTR allocation remains feasible. Thus, on the $Y$-axis in each graph, no FTRs are allocated, which reflects the initial situation as shown in Fig. 3, whereas on the $X$-axis in each graph, the FTRs to be allocated to market participants are represented up to their maximum feasible value. Furthermore, it is

\footnotetext{
15 Electricity cost of demand amount to 675 mEUR in the windy winter week. It represents the sum of hourly nodal load times nodal price. Thus, it can be interpreted as market volume.
}

important to note, that the distribution of FTRs differs for each week in the volume-based approach as the uniform pricing production in the week is used as distribution measure. For the capacity-based approach the distribution is constant as the installed generation capacities are the same.

Fig. 4 shows that both FTR distribution approaches yield qualitatively comparable results. The FTR allocation yields the expected benefit of reducing the distributional impact of the introduction of nodal pricing. The calculations also illustrate that both allocation regimes can only reduce the changes in surplus, but cannot precisely compensate all market participants. Comparing both allocation regimes, the volume-based approach achieves a favorable distribution for all three weeks. ${ }^{16}$ This can be attributed to the fact that the generation of the uniform pricing regime is taken as proxy for FTR allocation and thus varies between

\footnotetext{
16 We focus our analysis on replicating existing distributions of the uniform pricing setting. Therefore, surplus changes of all market participants are considered equally. Alternatively, minimizing surplus changes or final costs for specific market participants (e.g. load) are other options to select favorable allocation schemes.
} 


\section{No FTR allocation}

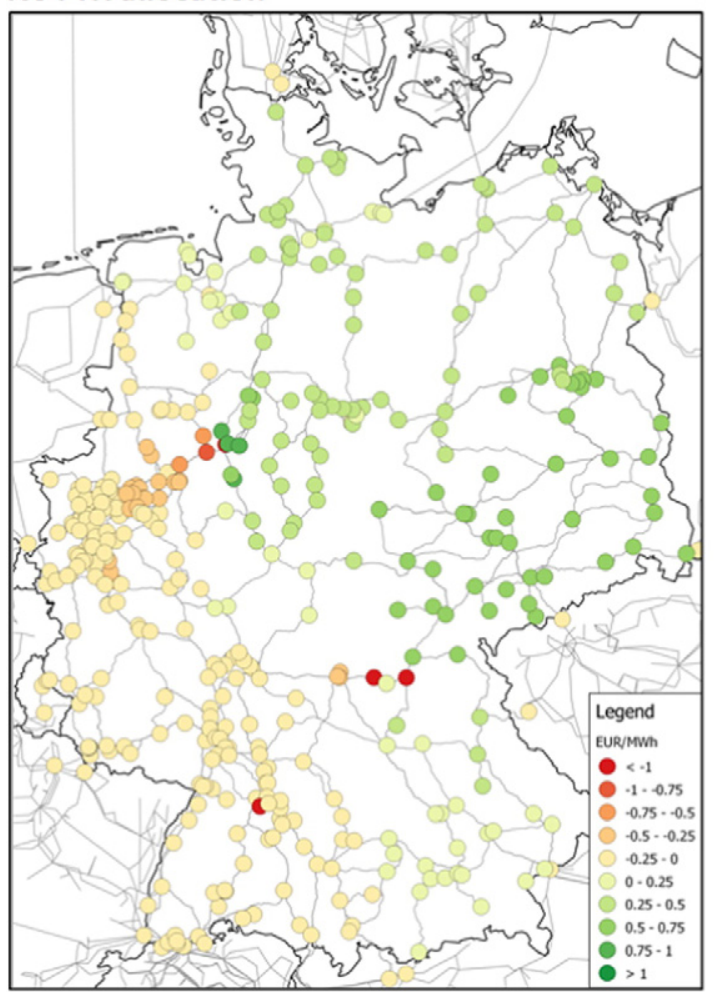

Full FTR allocation

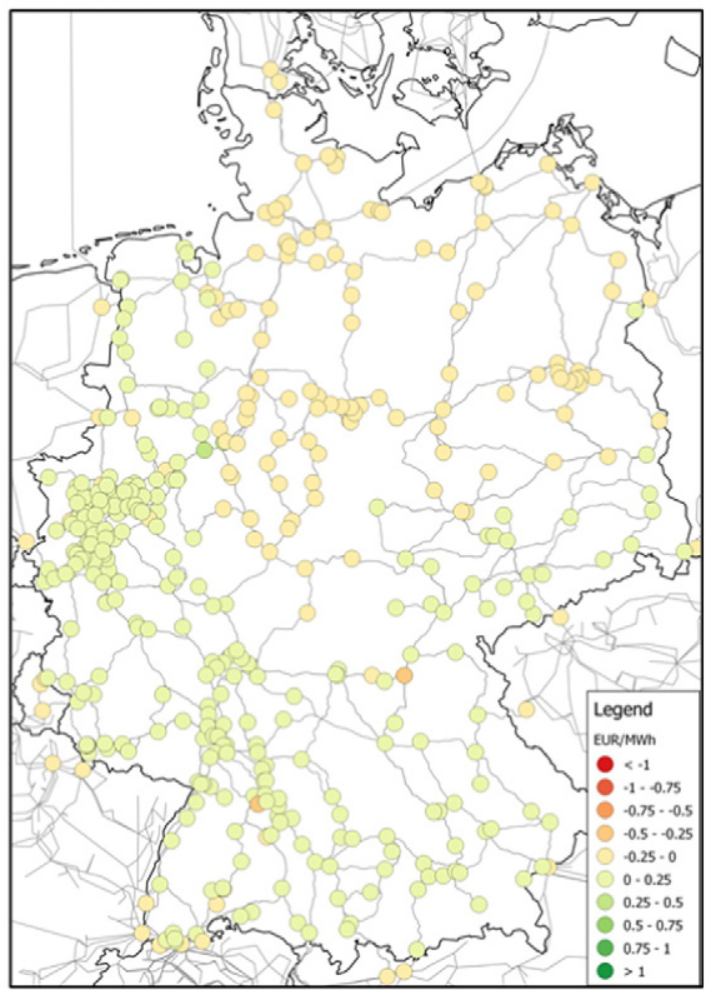

Fig. 5. Average change in surplus of demand in the high-wind winter week under volume-based allocation approach.

the three weeks. Thus, the low production of renewable generation in the first winter week is considered in the FTR allocation yielding in a higher allocation to conventional generation. In contrast, the share of FTRs allocated to renewable generation in the windy winter week is higher than conventional generation due to a higher production share. The FTR allocation in the capacity-based approach is, by definition, constant for all three weeks. Thus, if renewable production is low and the price at the nodes of renewable generation is below the reference value (e.g. because of large conventional generation), the negative value of FTRs reduces the surplus. ${ }^{17}$ The scale of the overall effect is small, but illustrates the challenge of using FTR obligations to hedge the output of intermittent renewable sources. FTR options would, in principle, avoid negative payments, but do not allow for netting as in the case of FTR obligations and thus reduce the volume of obligations that can be allocated.

Additionally, as both regionally diversified generation and load structure characterize the German power system, a closer look at the regional level is necessary in order to analyze the effectiveness of the allocation regimes. On an aggregated level, as presented here, the results are promising and may provide an argument to switch from the pricing regime toward nodal pricing.

In the following, we analyze the results at the regional level for load, conventional, and renewable generation. We focus our analysis on the winter week with high wind as it shows the greatest level of congestion among all considered weeks. Fig. 5 depicts the average change of demand's surplus at the regional level, differentiated between the initial situation without any FTR allocation (left side) and the situation with full volume-based FTR allocation (right side). As shown, congestion within the transmission network divides Germany into two parts: an

\footnotetext{
17 As renewable generation faces costs from FTR obligations, the congestion rent increases accordingly. Through socialization of the remaining congestion rent (initial congestion rent minus cost from FTR obligations), demand faces a positive effect in the winter week with low wind.
}

export constrained north-eastern part with lower average prices and an import-constrained south-western part with higher average prices. This development could be somewhat altered should solar deployment volumes in the south increase.

Henceforth, demand in the export-constrained region profits from lower electricity prices, whereas other regions face higher electricity costs and lose from a change in the pricing regime. Through an allocation of FTRs, as depicted on the right side of Fig. 5, demand in south-western Germany is compensated for the loss and vice versa for the benefits in north-eastern part. Through the allocation of FTRs, the changes in surplus resulting from the introduction of nodal pricing is significantly reduced.

To assess this effect in greater detail, Figs. 6 and 7 depict histograms of average surplus changes, differentiated by market participants considered. Fig. 6 presents the initial situation, which is characterized by high variations of participant's surpluses; in particular of demand and renewable generation.

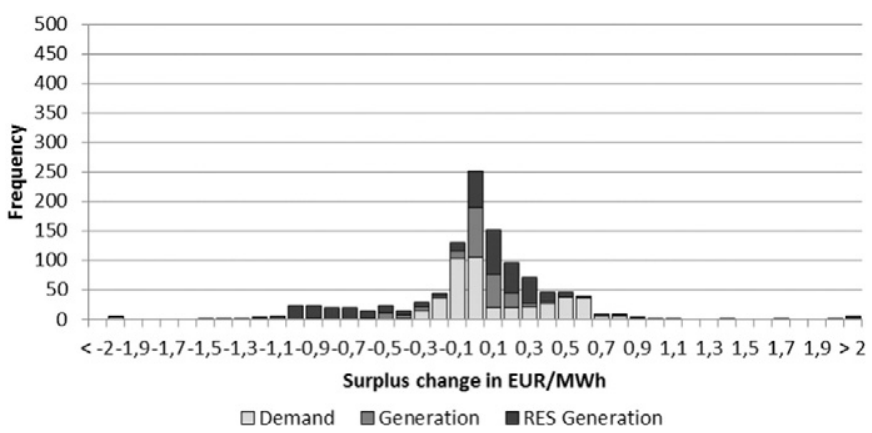

Fig. 6. Histogram of average nodal surplus changes in the high-wind winter week without FTR allocation. 


\section{Capacity-based FTR allocation}

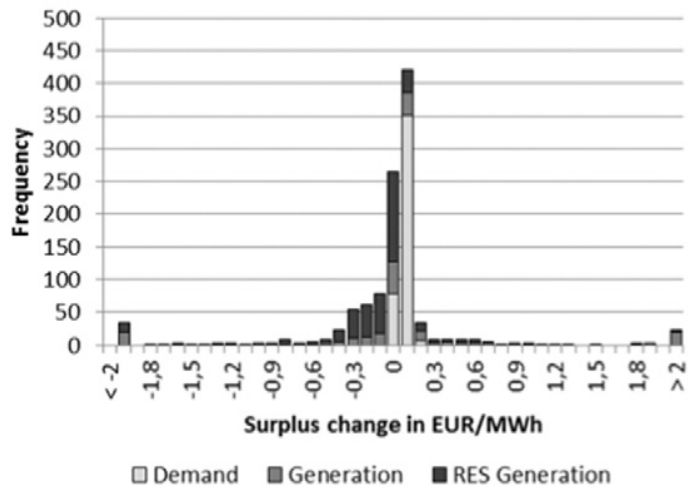

\section{Volume-based FTR allocation}

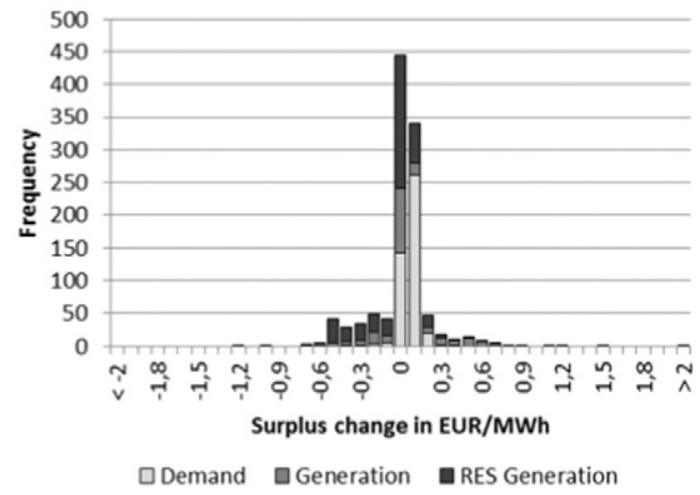

Fig. 7. Histogram of average nodal surplus changes in the high-wind winter week with capacity-based (left side) and volume-based FTR allocation (right side).

As shown in Fig. 7, the demand side with the allocation of FTRs can compensate for the surplus changes, thus mitigating the impact of introducing nodal pricing under either allocation methodology.

In the case of generation (both renewable and conventional), an allocation based on installed capacity can result in instances of significant increase or decrease of surplus (exceeding 2 Euro/MWh). This happens when generation is located at nodes with significant price changes and at the same time production volume is significantly below installed capacity. Thus, the effect only occurs in the capacitybased allocation method. Furthermore, the effect is linked to specific network circumstances: one generator in a generation pocket. Thus, we need to further assess the numerical results to assess to what extent this effect is material or a result of model calibration so as to inform a debate about implications for allocation methodologies or other issues, such as local market power mitigation procedures.

However, practically, wind power output will deviate in any hour from historic production volumes and, therefore, additional (hourly) variations must be anticipated, which will not necessarily balance out across the year. This again points to the difficulties of using FTR obligations as hedging instruments for wind power generation.

Fig. 8 depicts the standard deviation of surplus changes as a function of the volume of FTR allocation. It confirms the point that for demand and conventional generation, the allocation of FTRs can reduce most of the surplus change linked to the introduction of nodal pricing. It also illustrates that in case of conventional generation, the maximum allocation of FTRs results in an overcompensation of generation and, thus, the optimal volume of FTR allocation is below the maximum possible allocation. This result is linked to the use of FTR obligations that allow

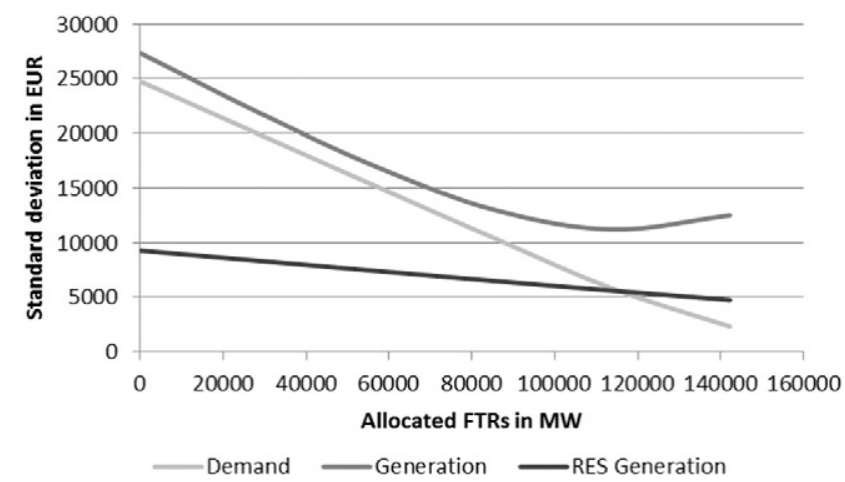

Fig. 8. Standard deviation of market participant's surplus in volume-based approach in the high-wind winter week (based on demand and generation at each node). for netting and, consequently, for a larger overall volume of FTRs to be allocated.

For renewable generation assets, the allocation of FTR obligations reduces only about half the standard deviation of surplus changes linked to nodal pricing introduction. Instead, if FTR options were allocated, then the allocation can only increase and not decrease the surplus. It also would result in a significantly lower volume of FTRs available for allocation, as netting is not possible, thus reducing the ability to compensate any of the market participants.

The presented analysis is based on two main assumptions which are of relevance in the context of FTRs. First, we fully abstract in our current modeling setting from transmission losses as we assume a lossless DC load flow approach. Accounting for transmission losses impacts in particular the locational prices in the nodal pricing system and thus can have implications on the effectiveness of the FTR allocation. Harvey and Hogan (2002) describe different design options for defining FTRs to hedge transmission losses. As we neglect transmission losses in the FTR allocation, revenue adequacy may be violated and the transmission system operator would face additional costs for compensating these transmission losses. Within our setting, these costs could then be socialized through network charges as it is currently applied in the German market. However, future research could extend our modeling setting by transmission losses as abstracting from transmission losses in a nodal pricing system may also impact the efficiency of the dispatch.

Secondly, we assume a fixed network topology and neglect any unavailability of transmission assets through, e.g. contingencies. Such unavailabilities would yield a derated transmission capacity and may violate the revenue adequacy of the FTR allocation. Thus, the transmission system operator is left with an imbalance of FTR revenues and costs. While this creates incentives for TSOs to schedule transmission maintenance at times of low congestion levels and to execute maintenance quickly, the associated risks may increase financing costs for TSOs. In the German system, the revenue shortfall could also be socialized through network charges as it is currently applied for congestion costs in the uniform pricing regime. ${ }^{18}$

Furthermore, our results and modeling approach rely on a perfectly competitive electricity market and we abstract from any strategic behavior of market participants. Strategic behavior can be present in the uniform pricing setting with a subsequent congestion management.

\footnotetext{
18 Hogan et al. (2010) additionally point out that a normal security-constrained, economic dispatch in electricity markets identifies in advance monitored contingencies and applies "n-1" limits so that the actual dispatch would remain feasible in the event of any of the monitored contingencies. Hence, for such contingencies, there is no dichotomy between normal dispatch and the contingency constrained dispatch. Normal "congestion" costs include the economic cost associated with the contingency. That is, all economic costs are congestion costs covered by the FTRs.
} 
If congestion management capacities are contracted through a separate bid- or market-based approach, incentives exist to profitably deviate from a competitive behavior in the congestion management market. In the context of the presented volume-based FTR allocation, market participants could then alter their behavior strategically to impact the FTR allocation. However, congestion management capacities in Germany are not contracted through a separate market but they are rather administratively required to participate in the congestion management and thus incentives for strategic behavior are rather limited. ${ }^{19}$ This assumption may not be valid for other markets or regions and may reduce the effectiveness of the proposed FTR allocations.

Further work is therefore needed to better explore the effectiveness in other regions and alternative FTR designs. The current application and the identified effects of the proposed FTR allocations are evaluated for the German system and an extension to other regions with differing spatial and technological characteristics is needed to verify the robustness of the achieved results. Regarding alternative FTR designs, these could involve splitting of FTR options so as to, for example, compensate renewable generation for the price difference up to a strike price at the reference node (Newbery and Neuhoff, 2008; Biggar and Hesamzadeh, 2013).

As of 2015, a more pragmatic solution is prominent in the US. Utility companies run tenders for renewable generation that offer a long-term off-take contract at the node of the generation asset. In selecting the winning bidder the utilities can consider congestion costs for delivering the energy to their customers, thus contributing to efficient locational choices, while the transmission price risk is pooled within a larger portfolio of utility and not imposed on project financed renewable projects. Feed-in tariffs typically offer a similar long-term off-take contract at the point of production, thus avoiding exposure of renewable projects to locational price risks. A new paradigm suggests that such feed-in tariffs should be replaced by (floating) market premium systems. Floating premiums provide a payment between an average system price and a strike price, but leave the renewable project investor exposed to any congestion risk linked to introduction of zonal or nodal pricing. This was not considered to be a relevant risk by the proponents of such a transition as they typically assume the feasibility of a single pricing zone at country level or beyond.

\section{Conclusions}

A major challenge for the adjustment of pricing zones and for the implementation of nodal pricing is the distributional impact of price changes facing generation and load in different locations of the system. The sum of changes to profits by individual generators and changes to costs faced by individual load of the price changes are typically several times higher than the efficiency savings delivered by an efficient congestion pricing mechanism while the benefits of such a change with respect to more reliable system operation are usually not ex-ante monetized.

In order to ensure that all (or most) parties benefit from and support the initial shift to an improved congestion management system before system security risks of inappropriate congestion management become apparent, it may be considered to mitigate the distributional impact. This may built on the experience that the implementation of nodal pricing is often accompanied with the free allocation of FTRs to market participants in proportion to their prevailing trading relationships (contracts or transfers within vertically integrated utilities) with the objective of mitigating the distributional impact. However, as in the European context most long-term contracting arrangements between

\footnotetext{
19 Generation capacities with a capacity above $10 \mathrm{MW}$ are required to be available for congestion management depending on their spot market commitments. Furthermore, the compensation of generators for positive redispatch and the payments from generators for negative redispatch are based on historical spot market prices to proxy generation costs.
}

generation and load have expired, the FTR allocation would have to be based on some other methodology instead.

Given the very political nature of rent allocation, the allocation methodology would have to be clearly specified and transparent. The purpose of this paper is to explore possible allocation alternatives, their representation in numerical models and their relative merits based from the model results.

In a three-node network, we find that allocation in proportion to historic annual production volume allows for better compensation of the distributional impact than allocation in proportion to installed capacity. This effect is dominated by the large discrepancy between available and used capacity of one generator in our stylized model. Furthermore, this highlights that the spatial and technological characteristics of the electricity system severely impact the effectiveness of the FTR allocations.

Hence, we explore the situation in a more realistic setting, modeling the FTR allocation for the German power system based on full nodal representation capturing its spatial and technological characteristics. For these specific circumstances, our results show that FTR allocation can mitigate almost all distributional effects for the demand side and a large share of the distributional effects for conventional generation. For intermittent renewables, the allocation of FTR obligations can mitigate fewer of the distributional effects, as the allocation profile will not match generation and could, at times, also increase the surplus change linked to the introduction of nodal pricing. This points to the need of either more complex FTR designs (option contracts), contractual arrangements (long-term off-take contracts) or legal frameworks (fixed feed-in tariffs) that insulate revenue streams from the introduction of additional zones or nodal pricing.

\section{Appendix A. Supplementary data}

Supplementary data to this article can be found online at http://dx. doi.org/10.1016/j.eneco.2016.09.018.

\section{References}

Adamson, S., Parker, G., 2013. Participation and Efficiency in the New York Financial Transmission Rights Market. In: Rosellón, J., Kristiansen, T. (Eds.), Financial Transmission Rights: Analysis, Experiences and ProspectsLecture Notes in Energy 7. Springer Verlag (ISBN: 978-1-4471-4786-2).

Biggar, D., Hesamzadeh, M.R., 2013. Designing Financial Transmission Rights to Facilitate Hedging in Wholesale Electricity Markets. 10th International Conference on European Energy Market, Stockholm, Sweden, 28-30 May 2013.

De Maere d'Aertrycke, G., Smeers, Y., 2013. Transmission Rights in the European Market Coupling System: An Analysis of Current Proposals. In: Rosellón, J., Kristiansen, T. (Eds.), Financial Transmission Rights: Analysis, Experiences and ProspectsLecture Notes in Energy 7. Springer Verlag (ISBN: 978-1-4471-4786-2).

Egerer, J., Gerbaulet, C., Ihlenburg, R., Kunz, F., Reinhard, B., von Hirschhausen, C., Weber A., Weibezahn, J., 2014. Electricity sector data for policy-relevant modeling: data documentation and applications to the German and European electricity markets. DIW Data Doc. 72 (http://www.diw.de/documents/publikationen/73/diw_01.c. 440963.de/diw_datadoc_2014-072.pdf).

Harvey, S.M., Hogan, W., 2002. Loss Hedging Financial Transmission Rights. http://www. hks.harvard.edu/fs/whogan/Harvey_Hogan_Loss_Hedging\%20_FTRs_011502_.pdf.

Hogan, W.W., 1992. Contract networks for electric power transmission. J. Regul. Econ. 4, 211-242.

Hogan, W.W., 2013. Financial Transmission Rights: Point-to Point Formulations. In: Rosellón, J., Kristiansen, T. (Eds.), Financial Transmission Rights: Analysis, Experiences and ProspectsLecture Notes in Energy 7. Springer Verlag (ISBN: 978-1-4471-4786-2).

Hogan, W., Rosellón, J., Vogelsang, I., 2010. Toward a combined merchant-regulatory mechanism for electricity transmission expansion. J. Regul. Econ. 38 (2), 113-143.

Kunz, F., 2013. Improving congestion management: how to facilitate the integration of renewable generation in Germany. Energy J. 34 (4), 55-78.

Leuthold, F., Weigt, H., von Hirschhausen, C., 2012. A large-scale spatial optimization model of the European electricity market. Netw. Spat. Econ. 12 (1), 75-107.

Newbery, D., Neuhoff, K., 2008. Market design for large shares of renewables: time and space, EPRG conference presentation. http://www.eprg.group.cam.ac.uk/wpcontent/uploads/2008/12/newbery-neuhoff.pdf.

NZEA, 2010. Managing locational price risk: proposal. Consultation paper at: https:// www.ea.govt.nz/dmsdocument/8139.

Olmos, L., Neuhoff, K., 2006. Identifying a balancing point for electricity transmission contracts. IEEE Trans. Power Syst. 21 (1), 91-98.

Read, E.G., 2007. Network congestion and wholesale electricity pricing in the Australian National Electricity Market: an analytical framework for describing options, EGR 
Consulting Ltd., report to AEMC. http://www.aemc.gov.au/getattachment/ d6aa8f5d-cce4-4ffd-9e31-a54c3e6e40c9/Network-Congestion-and-WholesaleElectricity-Prici.aspx.

Read, E.G., 2009. Locational hedging options for New Zealand: issues and options. EGR Consulting Ltd, report to NZEA. https://www.ea.govt.nz/dmsdocument/976.

Read, E.G., Jackson, P.R., 2013. Experience with FTRs and Related Concepts in Australia and New Zealand. In: Rosellón, J., Kristiansen, T. (Eds.), Financial Transmission Rights:
Analysis, Experiences and ProspectsLecture Notes in Energy 7. Springer Verlag (ISBN: 978-1-4471-4786-2).

Rosellón, J., Kristiansen, T. (Eds.), 2013. Financial Transmission Rights: Analysis, Experiences and ProspectsLecture Notes in Energy 7. Springer Verlag (ISBN: 978-14471-4786-2). 